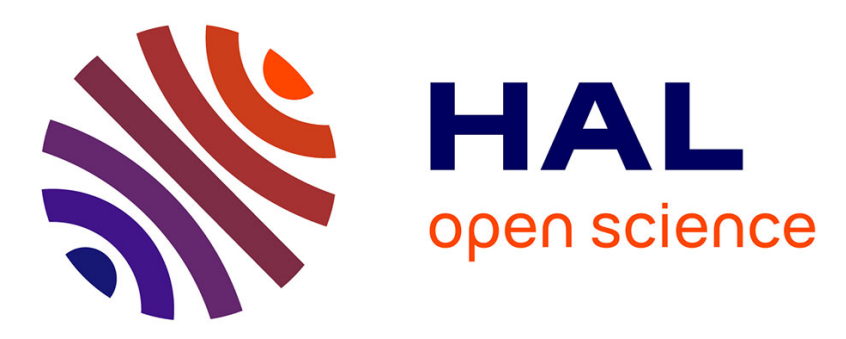

\title{
Transonic buffet instability: From two-dimensional airfoils to three-dimensional swept wings
}

\author{
Edorado Paladini, Samir Beneddine, Julien Dandois, Denis Sipp, \\ Jean-Christophe Robinet
}

\section{To cite this version:}

Edorado Paladini, Samir Beneddine, Julien Dandois, Denis Sipp, Jean-Christophe Robinet. Transonic buffet instability: From two-dimensional airfoils to three-dimensional swept wings. Physical Review Fluids, 2019, 4 (10), pp.103906. 10.1103/PhysRevFluids.4.103906 . hal-02455164

\author{
HAL Id: hal-02455164 \\ https://hal.science/hal-02455164
}

Submitted on 25 Jan 2020

HAL is a multi-disciplinary open access archive for the deposit and dissemination of scientific research documents, whether they are published or not. The documents may come from teaching and research institutions in France or abroad, or from public or private research centers.
L'archive ouverte pluridisciplinaire HAL, est destinée au dépôt et à la diffusion de documents scientifiques de niveau recherche, publiés ou non, émanant des établissements d'enseignement et de recherche français ou étrangers, des laboratoires publics ou privés. 


\title{
Transonic buffet instability: From two-dimensional airfoils to three-dimensional swept wings
}

\author{
Edorado Paladini, ${ }^{*}$ Samir Beneddine, Julien Dandois, and Denis Sipp \\ DAAA, ONERA, Université Paris Saclay, 8 rue des Vertugadins, 92190 Meudon, France \\ Jean-Christophe Robinet 18 \\ DynFluid Laboratory, Arts et Métiers Paris, 75013 Paris, France
}

The objective of the present study is to explain the evolution of the transonic buffet phenomenon from two-dimensional airfoils to three-dimensional swept wings by a global stability analysis. With respect to two-dimensional buffet, shock oscillation frequency increases by a factor of 4 to 7 in the case of a swept $30^{\circ}$ wing and three-dimensional patterns in the detached boundary layer are convected outboard. Crouch et al. [J. Comput.

Phys. 224, 924 (2007)] explained the two-dimensional transonic buffet phenomenon by the appearance of a real positive complex eigenvalue of the linearized Jacobian matrix. In the case of an infinite unswept wing, the present study shows that two unstable modes actually exist: The two-dimensional transonic buffet mode already identified by Crouch et al. [J. Fluid Mech. 628, 357 (2009)] and a strongly amplified three-dimensional zero-frequency mode. The latter exhibits regular patterns in the separated boundary layer, which relates to the so-called buffet cells as named by Iovnovich et al. [AIAA J. 53, 449 (2015)]. The nonzero sweep angle generates a spanwise velocity component on the wing which convects the cells outboard. This impacts both modes identified in the unswept case: The two-dimensional mode is weakly damped by the sweep while the three-dimensional buffet cells mode, even if weakly damped, remains strongly unstable and now exhibits a nonzero frequency which increases with the sweep angle. The frequency and wavelength of the most unstable three-dimensional mode for a sweep angle of $30^{\circ}$ agree well with numerical and experimental values of the three-dimensional transonic buffet on wings. The analysis of the wavemaker of the three-dimensional modes indicates that the core of the instability is nearly solely located in the separated region, with a maximum along the separation line. In contrast, the wavemaker of the two-dimensional buffet mode exhibits stronger values all along the

\section{INTRODUCTION}

Transonic buffet is an unsteady phenomenon which appears for certain values of Mach number $(M)$ and angle of attack $(\alpha)$. It consists in the oscillation of the shock on the suction side of a wing, synchronized with the thickening and thinning of the detached boundary layer. These oscillations modify the pressure field on the wing, inducing lift and drag oscillations. The result is an unwanted vibration of the wing, called buffeting, which in the worst case may cause structural damages due to fatigue. Consequently, the flight envelope of an aircraft in cruise flight is limited by transonic buffet.

\footnotetext{
*edoardo.paladini89@gmail.com
} 
Predicting the buffet onset and improving the understanding of the phenomenon is therefore a main concern for aircraft manufacturers.

Transonic buffet has been studied for many years over airfoils. It is now known that the phenomenon relates to a self-sustained feedback mechanism, which may be studied from two standpoints. On one hand, physical models, such that of Lee [1] and its variants proposed later by Hartmann et al. [2], Jacquin et al. [3], and Deck [4], describe well the phenomenon and have been numerically validated by several authors [4,5]. On the other hand, the existence of a self-sustained mechanism may be understood from a global stability analysis. The present work focuses on this second viewpoint. Crouch et al. [6,7] were the first to explain the transonic buffet phenomenon over two-dimensional (2D) airfoils by the appearance of an unstable global mode, i.e., a real positive complex eigenvalue of the linearized Jacobian matrix. From the study of this unstable mode, they proposed a model describing the self-sustained feedback loop based on perturbations generated at the shock foot that circumnavigate the shock. The global stability analysis of an airfoil was then repeated by Sartor et al. [8], who showed the complete spectrum at a fixed Mach number for increasing values of the angle of attack and identified the existence of a transonic buffet offset (the global mode becomes stable again).

Concerning the buffet phenomenon on three-dimensional (3D) wings, the first experimental tests were conducted by Roos [9]. He identified an unstable phenomenon linked with the oscillation of the shock wave, called the 3D transonic buffet. Reneaux et al. [10] and Molton et al. [11] defined precisely the differences in transonic buffet over airfoils and wings, in terms of spectral content of the unsteady pressure transducers and physics of the phenomenon. The shock oscillation has an amplitude about ten times smaller and a frequency four to seven times higher over wings than airfoils. Furthermore, the spectral content is a well-marked peak on airfoils while it is more broadband on wings. This suggests that the global transonic buffet mode is different between 2D airfoils and 3D wings. Dandois [12] and Koike et al. [13] gave typical values of the nondimensional shock oscillation frequency (Strouhal number $\mathrm{St}=f M A C / U_{\infty} \simeq 0.26$ ) and of the convection velocity of the phenomenon on the wing in the spanwise direction $\left(0.37 U_{\infty}\right)$, where $f$ is the frequency of the phenomenon in hertz, $M A C$ is the mean aerodynamic chord, and $U_{\infty}$ is the upstream velocity. They outlined the convective nature of the 3D mode. The 3D transonic buffet has been successfully simulated by both unsteady Reynolds-averaged Navier-Stokes (URANS) simulations [14,15] and detached-eddy simulation (DES) [16,17]. Iovnovich and Raveh [14] showed numerically the presence of 3D patterns convected outboard on a swept wing. These structures, called buffet cells, have been observed experimentally by Dandois [12] and Sugioka et al. [18]. Plante et al. [15] performed URANS simulations of the 3D transonic buffet for different sweep angles. In the unswept case, they observed an unstable steady phenomenon, which becomes unsteady when adding a sweep angle. The steady phenomenon exhibits buffet cells in the separated boundary layer. This shape is close to the shape of the physical phenomenon of stall cells which is better known at low speed, for instance, from the work of Rodriguez and Theofilis [19], who showed for incompressible flows that these cells originate from a 3D global unstable mode. The explication of the stall cells phenomenon by an unstable global mode was then questioned by a work of the same authors [20], where the mode appears to move towards unstable values but remains stable.

Yet, Iorio et al. [21], who performed a similar 3D analysis for the buffet phenomenon, did not find any 3D unstable modes. Similarly, Timme and Thormann [22] performed a 3D global stability analysis of the transonic buffet phenomenon on a realistic half-wing body configuration and only found a weakly damped global mode. It is only recently that Timme [23] found an unstable 3D mode on the same configuration but still did not answer the main question addressed in the present work: What is the link between 2D and 3D buffet? It is worth mentioning that the present work shares some similarities with that recently shown by Crouch et al. [24] in a conference. The main technical difference between our approach and theirs is that the analysis conducted by Crouch et al. uses a continuous Fourier representation for the spanwise homogeneous direction. Our approach is discrete in this direction and uses the method presented by Schmid et al. [25] to reduce the computational burden. While some conclusions are close, some findings of the present work significantly differ 


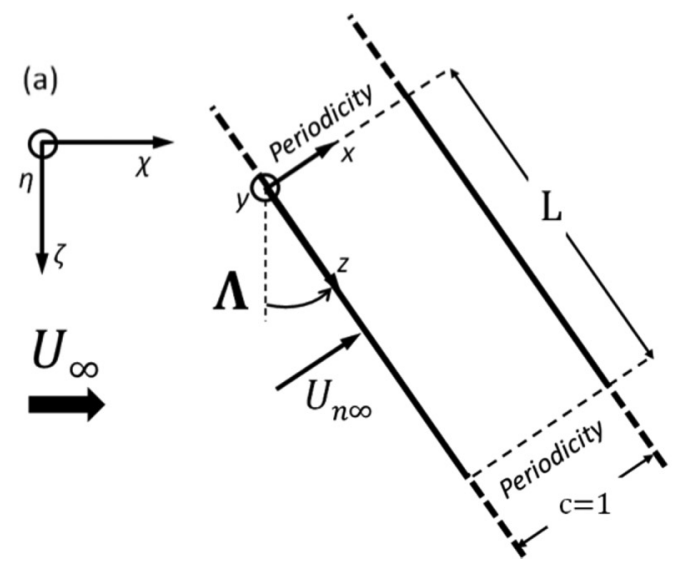

(b)

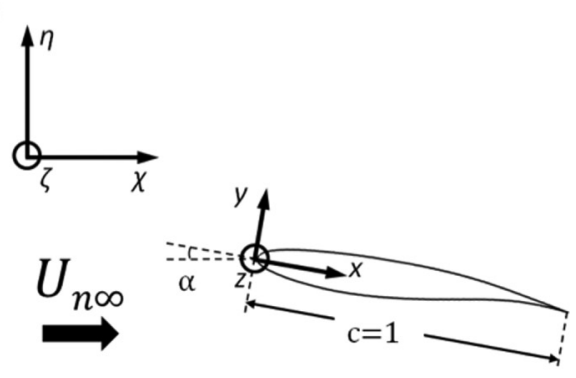

FIG. 1. Sketch of the infinite wing with geometrical variables and two reference frames: (a) top view for $\alpha=0^{\circ}$ and (b) side view for $\Lambda=0^{\circ}$.

from their results. Moreover, the present work goes further in the analysis by studying the adjoint modes and the sensitivity of the unstable modes.

The purpose of the present work is to answer three main questions by performing a global stability analysis of an infinite wing in the transonic regime. Does the stall cells unstable global mode observed in the incompressible regime also exist at transonic speeds? What is the effect of the sweep angle on the instabilities over an infinite wing? And, most important, what is the link between the 2D transonic buffet global mode and the so-called 3D transonic buffet?

The paper is organized as follows. In Sec. II, we first present the flow configuration, the governing equations, the numerical discretization techniques, and the resulting steady-state solutions. Then, Sec. III presents the global stability analysis framework and the numerical strategy to solve large $3 \mathrm{D}$ eigenproblems. The stability results of the paper are presented in Sec. IV: Mesh convergence, wavenumber, sweep angle, and angle-of-attack effects are detailed as well as the adjoint modes and structural sensitivity of the instabilities. Finally, Sec. V summarizes the main conclusions of the paper.

\section{FLOW CONFIGURATION, GOVERNING EQUATIONS, NUMERICAL DISCRETIZATION, AND BASE FLOW}

The airfoil geometry is ONERA's OAT15A transonic airfoil. Figure 1 shows a sketch of the flow configuration with the geometrical variables from two different viewpoints: From above the wing [Fig. 1(a)] and a side view [Fig. 1(b)]. The geometrical variables are the angle of attack (in the plane normal to the leading edge) $\alpha$, the sweep angle $\Lambda$, the chord $c$, and the spanwise extent $L$ of the computational domain. In the entire study, all lengths are made nondimensional with the chord. Two reference frames are shown in the figure: Relative to the upstream velocity, $R_{0}=(\chi, \eta, \zeta)$, and relative to the wing, $R_{w}=(x, y, z)$. The coordinate systems are the following: $\chi$ is oriented along the freestream direction, $\zeta$ is in the spanwise direction, and $\eta$ is vertical; $x$ is parallel to the airfoil chord, $z$ is parallel to the leading edge, and $y$ is perpendicular to the wing. In the reference frame $R_{0}$, the freestream velocity components along $\zeta$ and $\eta$ are zero, and the component along $\chi$ is denoted $U_{\infty}$. Figure 1 also introduces the velocity normal to the leading edge, $U_{n \infty}$, defined as $U_{n \infty}=\cos (\Lambda) U_{\infty}$. The origin of the reference frame $R_{w}$ is located at the airfoil leading edge as shown in Fig. 1 . In the following, the results are presented in the reference frame relative to the wing.

The governing equations are presented in a general conservative form as

$$
\frac{\partial \mathbf{q}}{\partial t}=\mathcal{R}(\mathbf{q})
$$




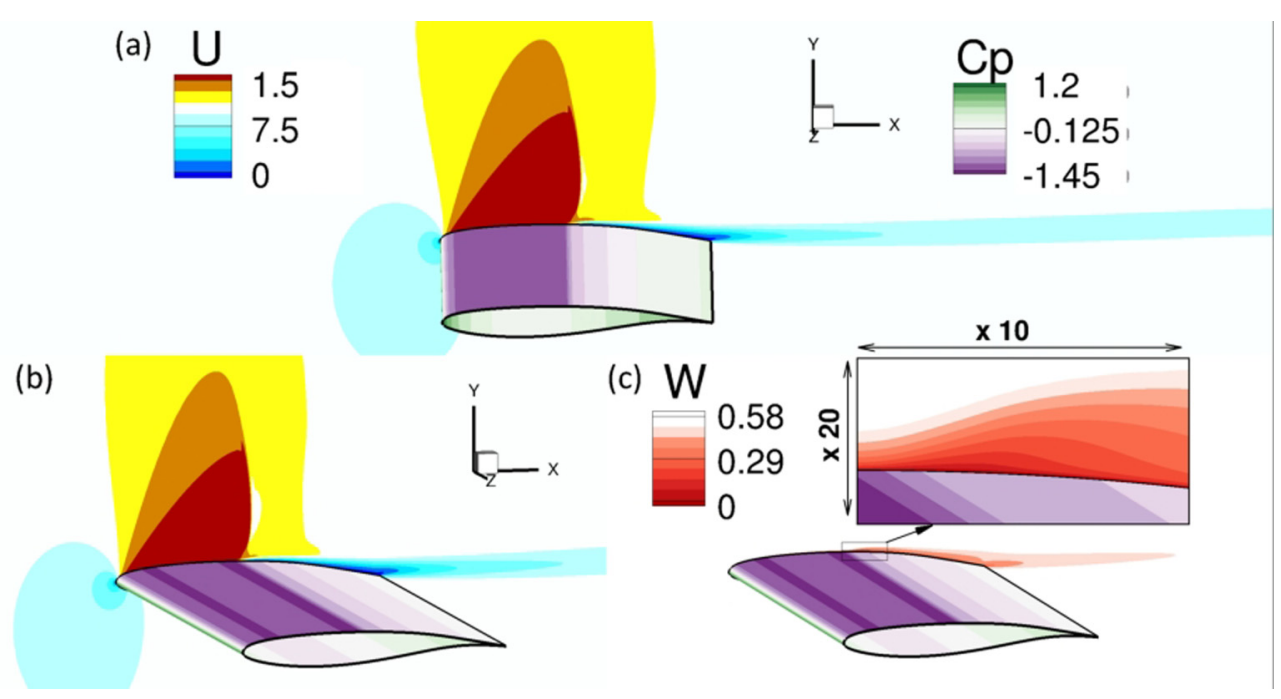

FIG. 2. Velocity fields in the $(x, y)$ plane of the wing reference frame and wall pressure coefficient $C_{p}$ for the 3D extruded base flow: (a) $U$ and $C_{p}$ for the unswept wing $\left(\Lambda=0^{\circ}\right.$ ), (b) $U$ and $C_{p}$ for the swept wing at $\Lambda=30^{\circ}$, and (c) spanwise component of the velocity, $W$, and $C_{p}$ for the swept wing at $\Lambda=30^{\circ}$ with a zoom at the shock foot. $\alpha=3.5^{\circ}$.

where $\mathcal{R}$ stands for the governing equations and $\mathbf{q}$ is the state variable. We consider compressible Reynolds-averaged Navier-Stokes (RANS) equations in conservative form closed with the SpalartAllmaras (SA) turbulence model [26] with the compressibility correction [27]. Hence, the state comprises the five conservative variables and one related to the turbulent eddy viscosity:

$$
\mathbf{q}=(\rho, \rho U, \rho V, \rho W, \rho E, \rho \tilde{v})^{T},
$$

where $\rho$ is the density, $(U, V, W)$ are the three components of the velocity $\mathbf{U}$ in the wing reference frame (see Fig. 1), $E$ is the total energy of the flow, and $\tilde{v}$ is the eddy viscosity of the SA turbulence model.

The numerical simulations are performed using the finite-volume solver FASTS from ONERA. The 3D Navier-Stokes equations are solved on multiblock structured grids. For the numerical method, a second-order AUSM+(P) upwind scheme [28] is used for the conservative variables and a first-order upwind scheme for the turbulent variable. For the time stepping, a first-order backward-Euler scheme is considered with local time stepping to obtain steady-state solutions. More details about the numerical method are available in [29].

In the following, all quantities are nondimensionalized by the infinite upstream variables of density $\rho_{\infty}$, the normal velocity $U_{n \infty}$, the total energy $E_{\infty}$, and the chord $c$.

Boundary conditions are adiabatic walls for the airfoil, a nonreflective boundary condition for the far-field 50 chord lengths away from the airfoil. The computational domain is discretized using a C-type structured grid. The mesh contains about $5 \times 10^{4}$ cells with a refinement around the timeaveraged shock location. The first mesh point in the boundary layer is below $y^{+}=1$.

The normal Mach number $M_{n \infty}$ in the $x-y$ plane (defined from the normal velocity component $U_{n \infty}$, i.e., $M_{n \infty}=M_{\infty} \cos (\Lambda)$, where $M_{\infty}$ is the freestream Mach number) is kept constant and equal to $M_{n \infty}=0.73$. The Reynolds number based on the chord length and the velocity $U_{n_{\infty}}$ is equal to $\operatorname{Re}_{c}=3.2 \times 10^{6}$. The sweep angle $\Lambda$ is simulated by imposing a constant spanwise velocity $W_{\infty}=U_{n \infty} \tan (\Lambda)$ in the reference frame of the wing at infinity. Figure 2 shows, for $\alpha=3.5^{\circ}$ and $\Lambda=0^{\circ}$ and $30^{\circ}$, the base flow $\mathbf{q}_{0}$, solution of $\mathcal{R}\left(\mathbf{q}_{0}\right)=0$, and in particular the boundary layer on the wing generated by $W_{\infty}$ in the swept case. 


\section{GLOBAL STABILITY ANALYSIS}

\section{A. Theory}

A linear stability analysis focuses on the evolution of a small amplitude perturbation $\mathbf{q}^{\prime}$ around a steady-state solution $\mathbf{q}_{0}$ :

$$
\mathbf{q}(x, y, z, t)=\mathbf{q}_{0}(x, y, z)+\mathbf{q}^{\prime}(x, y, z, t), \quad\left\|\mathbf{q}^{\prime}\right\| \ll\left\|\mathbf{q}_{0}\right\|,
$$

with $\|\cdot\|$ a given norm. In the following, the equations are first discretized and then linearized; the equations to the first order yield

$$
\frac{\partial \mathbf{q}^{\prime}}{\partial t}=J \mathbf{q}^{\prime}, \quad J_{k l}=\left.\frac{\partial \mathcal{R}_{k}}{\partial \mathbf{q}_{l}}\right|_{\mathbf{q}=\mathbf{q}_{0}},
$$

where $J$ is the linearized discrete Navier-Stokes operator $\mathcal{R}$ around the base flow. A normal mode analysis consists in considering solutions of the form

$$
\mathbf{q}^{\prime}(x, y, z, t)=\hat{\mathbf{q}}(x, y, z) \exp (\lambda t),
$$

where $\lambda$ is a complex quantity whose real part is the growth rate $\sigma$ of the perturbation and the imaginary part $\omega$ its angular frequency. $\hat{\mathbf{q}}(x, y, z)$ is the direct normal mode which represents the spatial coherent structure of the flow. Equation (4) then results in the direct eigenvalue problem:

$$
J \hat{\mathbf{q}}=\lambda \hat{\mathbf{q}}=(\sigma+i \omega) \hat{\mathbf{q}} .
$$

The adjoint eigenvalue problem associated with Eq. (6) is introduced in the following. Let us consider an inner product based on a real symmetric positive definite matrix $Q$ such that $\langle\mathbf{a}, \mathbf{b}\rangle=$ $\mathbf{a}^{*} Q \mathbf{b}$, where $\mathbf{a}$ and $\mathbf{b}$ are arbitrary vectors and the superscript * refers to the transconjugate. In the following the matrix $Q$ is chosen so that the induced norm verifies

$$
\|\left.\mathbf{q}\right|^{2}=\mathbf{q}^{*} Q \mathbf{q}=\iiint_{\Omega}\left(|\rho|^{2}+|\rho U|^{2}+|\rho V|^{2}+|\rho W|^{2}+|\rho E|^{2}+|\rho \tilde{\nu}|^{2}\right) d \Omega,
$$

where $\Omega$ is the computational domain. The adjoint matrix $J^{\dagger}$ is defined as the matrix satisfying

$$
\langle\mathbf{a}, J \mathbf{b}\rangle=\left\langle J^{\dagger} \mathbf{a}, \mathbf{b}\right\rangle \text {. }
$$

It is straightforward to show that

$$
J^{\dagger}=Q^{-1} J^{*} Q
$$

It follows that the eigenvalues of $J^{\dagger}$ are the complex conjugates of those of $J$. Given an eigenvalueeigenvector pair $(\lambda, \hat{\mathbf{q}})$, the associated adjoint global mode is defined as

$$
J^{\dagger} \mathbf{q}^{\dagger}=\lambda^{*} \mathbf{q}^{\dagger}=(\sigma-i \omega) \mathbf{q}^{\dagger} .
$$

The direct and adjoint global modes form a biorthogonal basis with respect to the considered scalar product: If the eigenvalues and direct and adjoint global modes are expressed as $\left(\lambda_{i}, \hat{\mathbf{q}}_{i}, \mathbf{q}_{j}^{\dagger}\right)$, then one may show that $\left(\lambda_{i}^{*}-\lambda_{j}\right) \mathbf{q}_{i}^{\dagger *} Q \hat{\mathbf{q}}_{j}=0$. Hence, either $\lambda_{i}^{*}=\lambda_{j}$ and $\mathbf{q}_{i}^{\dagger *} Q \hat{\mathbf{q}}_{j} \neq 0$ or $\lambda_{i}^{*} \neq \lambda_{j}$ and $\mathbf{q}_{i}^{\dagger *} Q \hat{\mathbf{q}}_{j}=0$. Furthermore, the convection operator in the Navier-Stokes equations results in a non-normal Jacobian matrix [30]. Consequently, direct global modes propagate downstream and adjoint global modes upstream [31]. The physical meaning of the adjoint modes corresponds to regions of the flow that are sensitive to force, damping, or in general control the phenomenon [32]. They underline the region in the flow where a forcing results in the strongest effect on the unstable dynamics of the flow.

\section{B. Eigenmode computation strategy}

From a physical viewpoint, only the least-damped or unstable eigenvalues of Eqs. (6) and (10) are of interest. Therefore, rather than performing a full computation of the spectrum, a classical 
approach consists in using Krylov algorithms coupled with a shift-and-inverted strategy, yielding all eigenvalues in the neighborhood of a chosen shift [33]. For configurations with a reduced number of degrees of freedom, such as two-dimensional cases, the inversion stage may be performed using a direct lower-upper (LU) solver, but for 3D configurations, the memory cost of an LU factorization may become prohibitive. In all cases considered here, the base flow is homogeneous in the spanwise direction (see Sec. II), leading to a particular structure of the Jacobian matrix (and its adjoint), which makes the specific 3D eigenproblem computations considered here affordable.

To detail the method, let us now consider a discrete framework where the spanwise direction is regularly discretized, and where the indexing of the degrees of freedom is such that an eigenvector $\hat{\mathbf{q}}$ reads

$$
\hat{\mathbf{q}}=\left(\hat{\mathbf{q}}_{0}^{T}, \hat{\mathbf{q}}_{1}^{T}, \ldots, \hat{\mathbf{q}}_{N_{z}-1}^{T}\right)^{T}
$$

where $\hat{\mathbf{q}}_{i}$ is a block associated with the flow values of the $i$ th spanwise slice and $N_{z}$ is the number of points in the spanwise direction. The spanwise homogeneity of the flows makes any slice in this direction undifferentiated from the others, resulting in the following block-circulant structure of the Jacobian matrix:

$$
J=\left[\begin{array}{ccccc}
A_{0} & A_{1} & \cdots & A_{N_{z}-2} & A_{N_{z}-1} \\
A_{N_{z}-1} & A_{0} & \cdots & A_{N_{z}-3} & A_{N_{z}-2} \\
A_{N_{z}-2} & A_{N_{z}-1} & \cdots & A_{N_{z}-4} & A_{N_{z}-3} \\
\vdots & \vdots & \vdots & \vdots & \vdots \\
A_{1} & A_{2} & \cdots & A_{N_{z}-1} & A_{0}
\end{array}\right]
$$

where each column of blocks is associated with a given spanwise slice. One may then show that all eigenmodes are harmonic in the spanwise direction [25], which reads in a discrete framework

$$
\hat{\mathbf{q}}=\left(\hat{\mathbf{q}}_{0}^{T}, \rho_{j} \hat{\mathbf{q}}_{0}^{T}, \rho_{j}^{2} \hat{\mathbf{q}}_{0}^{T}, \ldots, \rho_{j}^{N_{z}-1} \hat{\mathbf{q}}_{0}^{T}\right)^{T},
$$

with $\rho_{j}=e^{j \frac{2 i \pi}{N_{z}}}\left(0 \leqslant j \leqslant N_{z}-1\right)$ a root of unity corresponding to a given spanwise wavenumber $\beta_{j}=2 j \pi / L$, where $L$ is the physical length of the computational domain in the spanwise direction. In this framework, as shown by Schmid et al. [25], all the eigenmodes of the full 3D system associated with a given wavenumber $\beta_{j}$ may be equivalently obtained by solving the reduced eigenvalue problem

$$
\hat{A}_{j} \hat{\mathbf{q}}_{0}=\lambda \hat{\mathbf{q}}_{0}, \quad \text { with } \hat{A}_{j}=\sum_{k=0}^{N_{z}-1} \rho_{j}^{k} A_{k},
$$

and then by using Eq. (13) to compute the full 3D vector. By an adequate choice of $L, N_{z}$, and $j$, one may then perform a 3D global stability analysis for some prescribed wavenumbers at the cost of a two-dimensional analysis. In particular, the numerical cost is independent of the spanwise discretization, which can be arbitrarily dense. With the chosen spatial five-point discretization scheme, which achieves second-order accuracy, only the matrices $A_{N_{z-2}}, A_{N_{z-1}}, A_{0}, A_{1}$, and $A_{2}$ are nonzero. Note that the same strategy may be followed to compute adjoint modes. The results presented in the following are based on the implicitly restarted Arnoldi method using the open source library ARPACK [34] to solve the reduced eigenproblem, coupled with the direct LU solver MUMPS [35]. The Jacobian matrix is obtained by a second-order finite differences linearization of the discrete RANS equations following the procedure detailed in [33].

\section{STABILITY RESULTS}

\section{A. 2D and 3D buffet modes: Case $\alpha=3.5^{\circ}, \Lambda=0^{\circ}, \beta=0,2 \pi, 4 \pi, 8 \pi$}

Let us first focus on an unswept wing $\left(\Lambda=0^{\circ}\right.$ ) at an incidence of $\alpha=3.5^{\circ}$ (the influence of these two parameters is studied in Secs. IV C through IV F). The global stability analysis reveals two types 


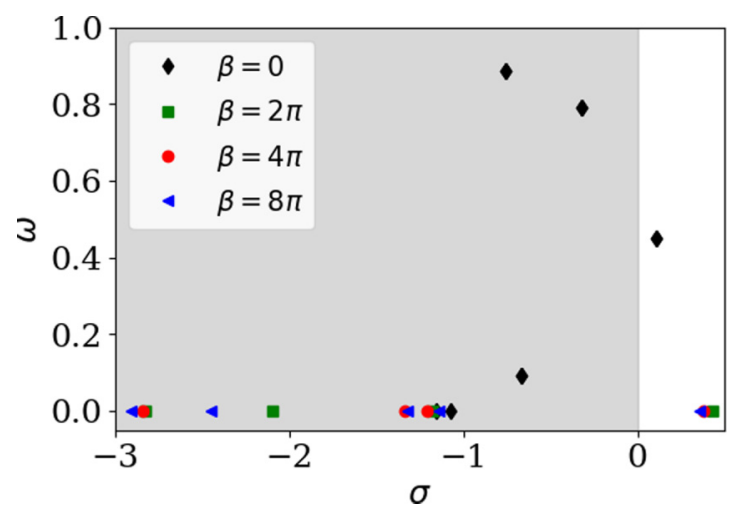

FIG. 3. Spectrum of the unswept $\left(\Lambda=0^{\circ}\right)$ wing for $\beta=0, \beta=2 \pi, \beta=4 \pi, \beta=8 \pi$, and $\alpha=3.5^{\circ}$. Unstable eigenvalues $(\sigma>0)$ are in the white half plane.

of unstable modes: An unsteady (non-zero-frequency) 2D mode $(\omega \neq 0, \beta=0)$ and several steady (zero-frequency) 3D modes $(\omega=0, \beta \neq 0)$. Figure 3 shows spectra for $\beta=0$ and a few nonzero $\beta$ values.

The 2D unsteady mode corresponds to the buffet mode found by Crouch et al. [6,7], Sartor et al. [8], and Iorio et al. [21] for a 2D airfoil. It exhibits a shock oscillation behavior synchronized with the thickening and thinning of the boundary layer [Fig. 4(a)]. This mode does not exhibit a spanwise component $[\hat{w}(x, y, z)=0]$. The angular frequency value $(\omega=0.45)$ is in agreement with numerical simulations [4,5] and experimental tests [1,3] of transonic buffet over 2D airfoils which give results in the range $\omega=0.41-0.48$.

The 3D unstable modes exist for a wide range of nonzero wavenumber $\beta$ and are steady $(\omega=0)$. Consequently, no spanwise propagating phenomenon appears on an unswept wing. Figure 4(b) shows the spatial structure of the mode for $\beta=2 \pi$, which is similar to those observed for other wavenumbers.

These 3D modes exhibit coherent structures located in the separated region and along the shock wave. Their structure in the $x-y$ plane, although similar to the $2 \mathrm{D}$ unstable mode, less affects the shockwave. The modes oscillate in the spanwise direction as the buffet cells observed in URANS simulations and DESs. The 3D modes exhibit a flow structure similar to the stall cell phenomenon in

(a)

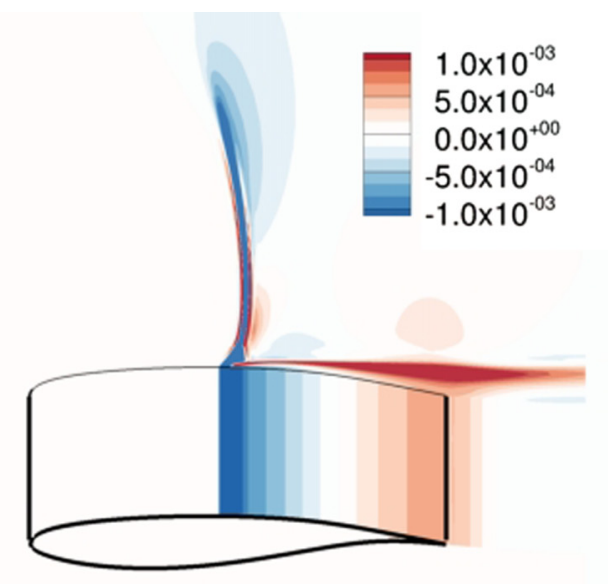

(b)

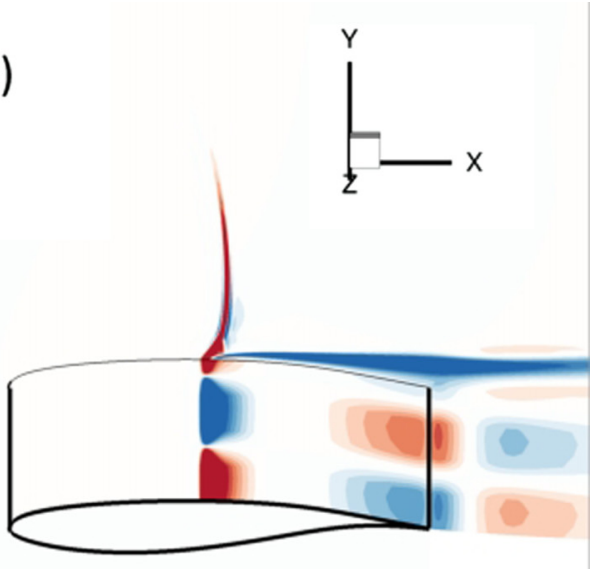

FIG. 4. Real part of the $\rho E$ component of (a) the 2D buffet mode ( $\omega=0.45)$ at $\beta=0$ and (b) the 3D buffet cells mode $(\omega=0)$ at $\beta=2 \pi$, respectively. 

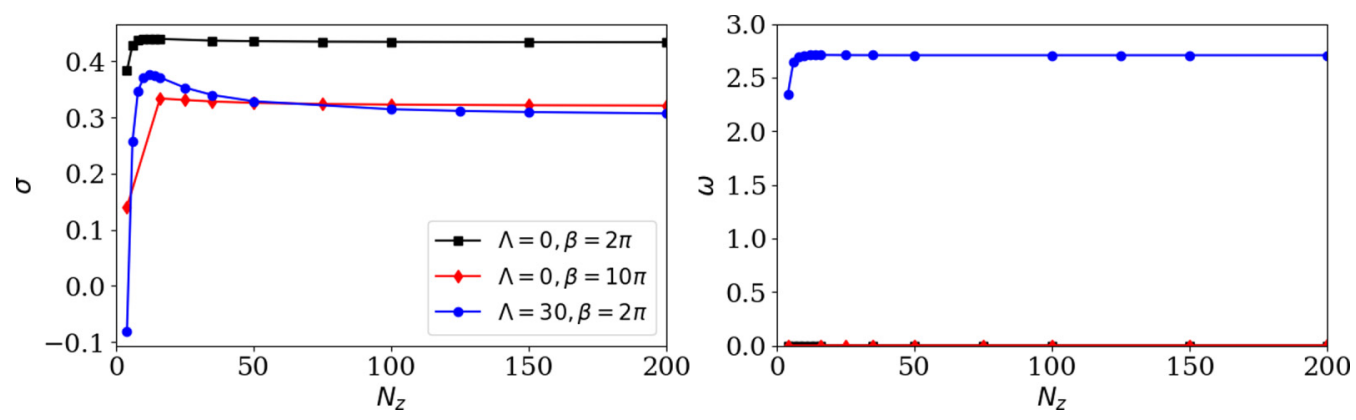

FIG. 5. Effect of the mesh refinement in span on the growth rate and frequency of the 3D mode for different wavenumbers $\beta$, sweep angles $\Lambda$, and $\alpha=3.5^{\circ}$.

the detached boundary layer [36]. Yet, the reasons behind the detachment of the boundary layer are different: At low speed the boundary layer at the suction side detaches because of the smooth and progressive recompression of the flow towards the trailing edge of the airfoil, while in the transonic regime the detachment results from the strong interaction of the boundary layer with the shock wave.

\section{B. Convergence study for the 3D buffet mode}

While the effect of the mesh refinement in the spanwise direction does not impact the 2D unstable mode, it has an important effect on the three-dimensional modes. Even though the method introduced in Sec. III B allows an arbitrarily dense discretization of the spanwise direction, one has to ensure that the chosen parameter $N_{z}$ provides converged results. This point has been addressed by computing the evolution of the eigenvalue associated with the unstable $3 \mathrm{D}$ mode with respect to $N_{z}$ for different values of $\beta$. Figure 5 shows the results for different wavenumber values for an unswept and a swept wing at $\Lambda=30^{\circ}$ (3D modes at $\Lambda \neq 0$ are presented in Sec. IV C), both for $\alpha=3.5^{\circ}$. The frequency converges quickly with respect to $N_{z}$ but the growth rate displays a more particular behavior: The convergence is not monotonic, and while a value as low as $N_{z}=12$ yields well-converged results sometimes (for instance for $\beta=2 \pi$ and $\Lambda=0^{\circ}$ ), in the worst cases observed, $N_{z}$ has to be higher than 100 to truly consider the result as converged. In the present paper, all stability results verify $N_{z} \geqslant 200$.

\section{Sweep effect for $\alpha=3.5^{\circ}, \beta=0$ and $\beta=2 \pi$}

This section focuses on the evolution of the 2D mode $(\beta=0)$ and the 3D mode for $\alpha=3.5^{\circ}$, when the sweep angle $\Lambda$ is varied. For the 3D mode, we only focus here on a fixed wavelength number $\beta=2 \pi$ (this specific choice is explained in Sec. IV D). Figure 6(a) shows the path followed by the two unstable eigenvalues as $\Lambda$ increases from $0^{\circ}$ to $30^{\circ}$. The $2 \mathrm{D}$ mode for the unswept wing undergoes a slight stabilization with $\Lambda$ while remaining almost at the same frequency. The growth rate decreases from 0.107 with no sweep to 0.08 at $\Lambda=30^{\circ}$. The structure of the mode does not change in the $x-y$ plane. For $\Lambda>0$, a spanwise component of the velocity $\widehat{\rho w}(x, y, z)$ appears; it is constant in span, so the mode remains strictly $2 \mathrm{D}$. In the case of a base flow which does not vary in span, the $\rho u$ and $\rho v$ dynamics are independent of that of $\rho w$. The variation of the eigenvalue (growth rate decrease) is due to a weak coupling which is present only in compressible flows and which stems from the diffusive term in the energy conservation equation $(\rho E)$. More precisely, the coupling is due to the term $\operatorname{div}(\Sigma \mathbf{U})$ in the energy equation, where div is the divergence in the 3D Euclidean space and $\Sigma$ is the strain tensor.

Regarding the 3D buffet mode, as the sweep angle departs from zero, the mode exhibits an oscillatory behavior $(\omega \neq 0)$. The frequency gradually increases with the sweep angle up to $\omega=2.7$ at $\Lambda=30^{\circ}$ (six times the $2 \mathrm{D}$ mode frequency). At the same time, the growth rate decreases from 
(a)

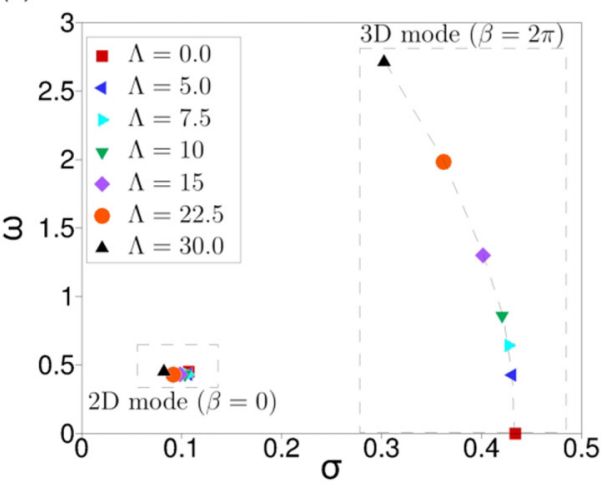

(b)

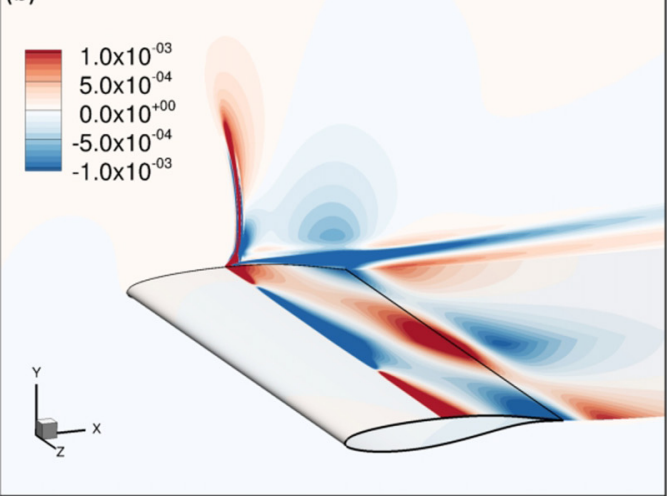

FIG. 6. (a) Evolution of the unstable eigenvalues for $\beta=0$ and $\beta=2 \pi$ for an airfoil with a sweep angle $\Lambda$ increasing from $0^{\circ}$ to $30^{\circ}\left(\alpha=3.5^{\circ}\right)$. (b) Real part of the $\rho E$ component of the $3 \mathrm{D}$ buffet mode at $\beta=2 \pi$, $\Lambda=30^{\circ}$, and $\alpha=3.5^{\circ}$.

0.43 to 0.30 at $\Lambda=30^{\circ}$. The buffet cell mode is therefore convected outboard with a convection velocity $U_{c}$ proportional to the sweep angle and the amplitude of $\widehat{\rho w}$ component of the mode increases. The outboard convection velocity of the $3 \mathrm{D}$ phenomenon can be computed as $U_{c}=\omega / \beta$. This convection velocity increases with the sweep angle, up to $0.43 U_{n \infty}$ (or $0.37 U_{\infty}$ ) for $\Lambda=30^{\circ}$, which is exactly the same value found experimentally by Dandois [12] and Koike et al. [13]. The results of the present study reveal that the ratio $c$ between this convection speed and the spanwise flow velocity $W_{\infty}=U_{n \infty} \tan (\Lambda)$ (defined as $c=U_{c} / W_{\infty}$ ) remains more or less constant for all sweep angles, with a value around 0.76. This is further discussed in Sec. IV D.

The flow structure of the 3D mode at $\Lambda=30^{\circ}$ is shown in Fig. 6(b). It is very similar to the unsteady structures of the flow found numerically by Iovnovich and Raveh [14] and Plante et al. [15] and experimentally by Dandois [12] and Sugioka et al. [18] for the 3D buffet. The evolution of the spectrum with the sweep angle suggests that the phenomenon known as 3D transonic buffet is not linked with the 2D one: There are two distinct unstable modes in the spectrum. The 3D buffet phenomenon at nonzero sweep angles corresponds to the steady global mode associated to buffet cells observed for an unswept wing, its frequency becoming nonzero because of the addition of sweep which convects the $3 \mathrm{D}$ structures outboard. It is justified in Sec. IV D why $\beta=2 \pi$ is the right wavenumber to pick for this comparison.

\section{Effect of wavenumber for $0^{\circ} \leqslant \Lambda \leqslant 30^{\circ}$ at $\alpha=3.5^{\circ}$}

In the present section, the effect of the spanwise wavenumber $\beta$ on the different unstable modes is studied for different values of $\Lambda$ and for $\alpha=3.5^{\circ}$. The analysis first reveals that the $2 \mathrm{D}$ buffet mode is quickly damped as $\beta$ increases to nonzero values: The critical values are $\beta \approx 0.9$ for the unswept wing and $\beta \approx 0.6$ for the swept wing at $\Lambda=30^{\circ}$ (Fig. 7). Nonetheless, the strongest growth rate is found at $\beta=0$, which confirms the 2D nature of the mode (Fig. 7). The spatial structure of the mode for $\beta \neq 0$ is, by definition, no longer $2 \mathrm{D}$, but it only exhibits a slight variation in span (see Fig. 8) and strongly resembles the purely two-dimensional buffet mode. Regarding the 3D mode, Figs. 9(a) and 9(b) show the variation of its growth rate and frequency with the wavenumber for different values of $\Lambda$ while keeping $\alpha=3.5^{\circ}$. The mode is stable for $\beta \lesssim 2$, which confirms its three-dimensional nature. Interestingly, for low sweep angles, the curves display two local maxima corresponding to two a priori distinct instabilities that appear in the flow: The already discussed 3D buffet mode at $\beta \approx 2 \pi$ and another $3 \mathrm{D}$ instability at $\beta \approx 7 \pi$. For the sake of conciseness, let us refer to these modes as low-wavenumber (LW) and high-wavenumber (HW) modes, respectively. 

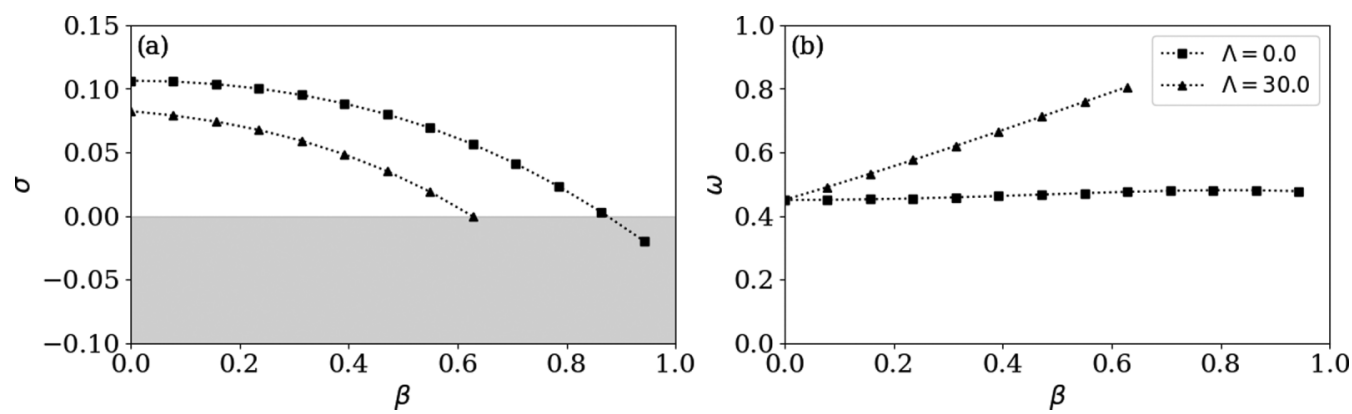

FIG. 7. Growth rate and frequency of the "2D" buffet mode as a function of the wavenumber for the unswept $\Lambda=0^{\circ}$ and swept $\Lambda=30^{\circ}$ wing, $\alpha=3.5^{\circ}$. Although unstable modes exist for $\beta \neq 0$, the most amplified wavenumber is $\beta=0$, hence the designation of the mode as a $2 \mathrm{D}$ instability.

The HW mode has also been observed by Crouch et al. [24]. In the present computations, and in contrast to the results obtained by Crouch et al., its existence strongly depends on the value of the sweep angle, and it even disappears for high sweep angles, the critical value being around $\Lambda=30^{\circ}$. But for all the cases considered in this section, the HW mode is not dominant, and the most unstable $3 \mathrm{D}$ buffet cell mode for all sweeps is the LW mode $(\beta \approx 2 \pi)$. This LW mode remains unstable as the sweep is increased, and the most amplified wavenumber is only weakly affected by the sweep angle. This is why all the previous spectra regarding the 3D mode have been mostly focused on the $\beta=2 \pi$ case. This dominant wavenumber value for the three-dimensional buffet mode is consistent with the results of Dandois [12] $(\beta=2.6 \pi)$, Iovnovich and Raveh [14] $(\beta=2.6 \pi)$, and Plante et al. [15] $(\beta=2.9 \pi)$. The variation of the frequency in the swept wing case is shown to be linear with the wavenumber, while it is zero for the unswept case [see Fig. 9(b)]. This is consistent with the conclusions from Sec. IV C, which showed that the convection speed $U_{c}=\omega / \beta$ was approximately equal to $c W_{\infty}$, with $W_{\infty}=\tan (\Lambda)$ (see Sec. II) and $c \approx 0.76$. A more accurate estimation of $c$ may be obtained by computing

$$
c=\underset{\tilde{c}}{\operatorname{argmin}}\left(\sum_{i}\left(\frac{\omega_{i}}{\tan \left(\Lambda_{i}\right) \beta_{i}}-\tilde{c}\right)^{2}\right),
$$

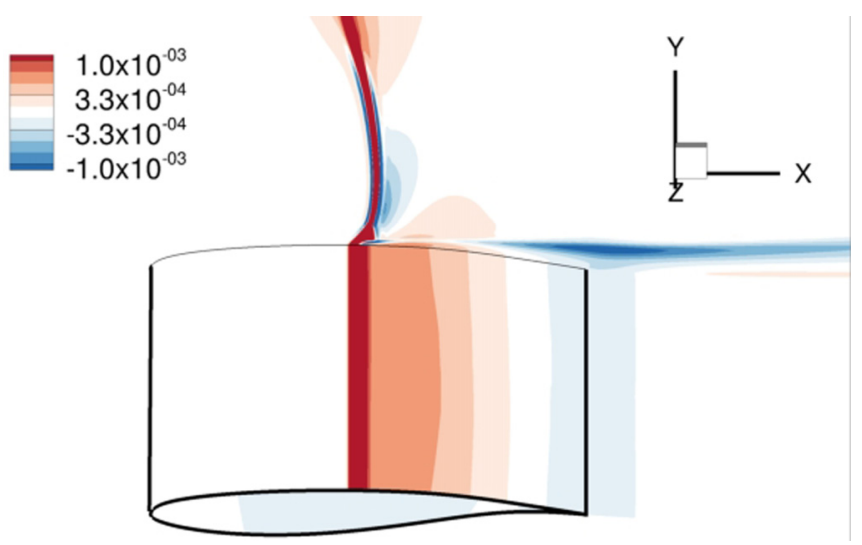

FIG. 8. Real part of the $\rho E$ component of the (quasi-)2D buffet mode for the unswept wing $\Lambda=0^{\circ}$, $\alpha=3.5^{\circ}$, and wavenumber $\beta=0.15 \pi$ (the mode is not $2 \mathrm{D}$ because $\beta \neq 0$, but the spanwise variations are slight). 

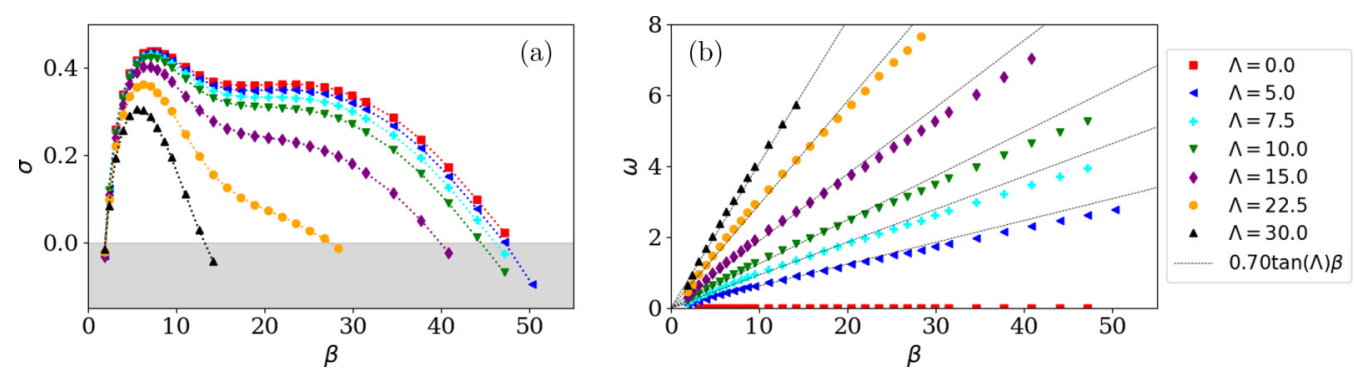

FIG. 9. Growth rate and frequency of the 3D mode as a function of the wavenumber for the unswept $\Lambda=0^{\circ}$ and swept wings $5^{\circ} \leqslant \Lambda \leqslant 30^{\circ}$. $\alpha=3.5^{\circ}$.

where $\omega_{i}, \Lambda_{i}$, and $\beta_{i}$ are all the data from Fig. 9 (except those for $\Lambda=0$ ). This may be computed using a gradient descent algorithm, which yields $c \approx 0.70$, in good agreement with the rough estimate of Sec. IV C.

A first comparison with the results given in [24] is provided here. Qualitatively, both studies are in good agreement: Two different $3 \mathrm{D}$ modes have been found, a LW mode around $\beta=2 \pi$ and a HW mode around $\beta \approx 30$, both modes being steady for $\Lambda=0^{\circ}$ and unsteady when $\Lambda \neq 0^{\circ}$. Moreover, the values of the growth rates for the LW 3D buffet mode at $\beta=2 \pi$ are very similar. But some quantitative differences appear with respect to the 3D HW mode. Around $\beta \approx 30$, Crouch et al. [24] reported values of growth rates approximately three times larger than at $\beta \approx 2 \pi$ and the stabilizing effect of the sweep angle for high-wavenumber perturbations appears much weaker in their work. In our study, the HW mode even disappears for $\Lambda>30^{\circ}$ while it remains strongly unstable in their paper. Yet, the two studies have been conducted on the same OAT15A airfoil, under the same flow condition, with the same angle of attack and same turbulence model, and both results are well spatially converged. Hence, only different numerical spatial discretization strategies may account for these discrepancies, while it is well known that buffet configurations are very sensitive to such choices.

These discrepancies are further discussed in the next section, which studies the effect of the angle of attack on the 3D modes.

\section{E. Effect of wavenumber for $3.0^{\circ} \leqslant \alpha \leqslant 4.5^{\circ}$ at $\Lambda=0^{\circ}$}

This section focuses on the unswept wing $\left(\Lambda=0^{\circ}\right)$ and analyzes the effect of the angle of attack on the most amplified wavenumber associated with the 3D mode. It aims at providing further insights with respect to the discrepancies highlighted in Sec. IV D concerning the HW mode between the present results and those of Crouch et al. [24]. Figure 10 shows the evolution of the

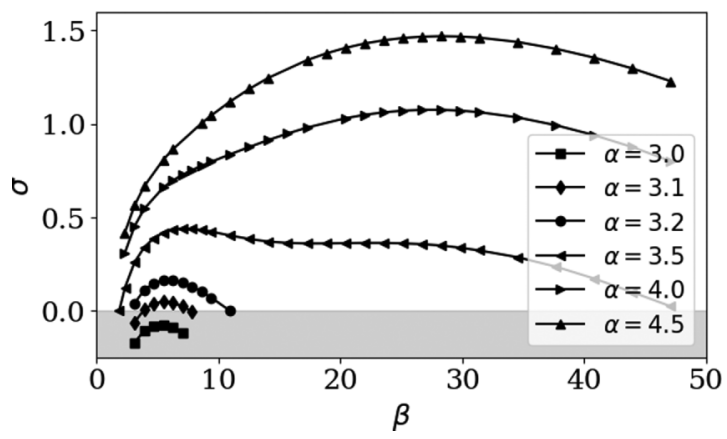

FIG. 10. Growth rate and frequency of the 3D mode on the unswept wing $(\Lambda=0)$ as a function of the wavenumber for different angle of attack $\alpha$. 

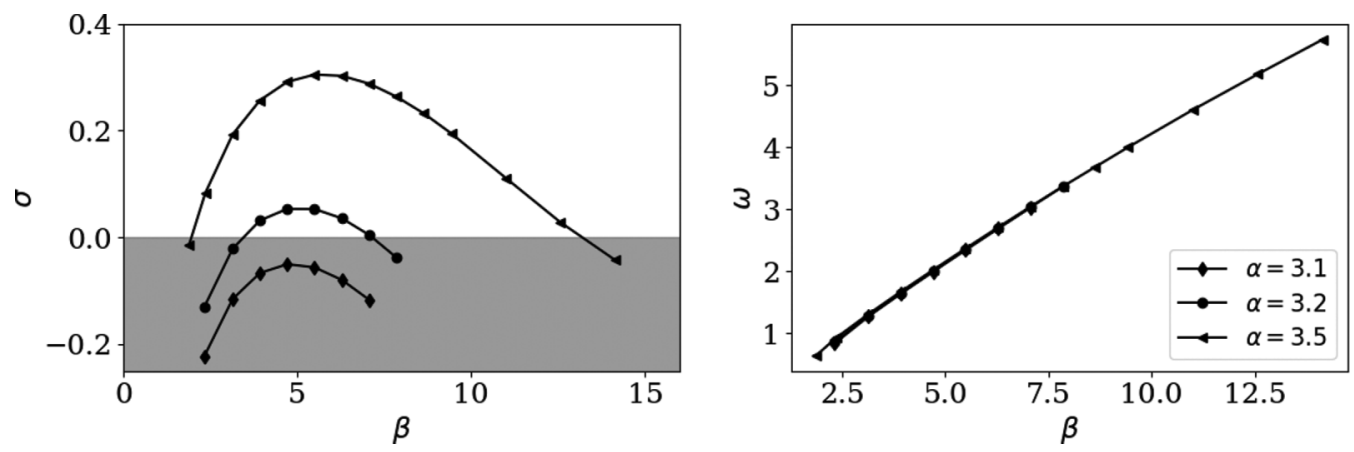

FIG. 11. Growth rate and frequency of the $3 \mathrm{D}$ mode on the unswept wing $\left(\Lambda=30^{\circ}\right)$ as a function of the wavenumber for different angle of attack $\alpha$.

growth rate of the 3D mode with respect to $\beta$ for different angles $\alpha$, including values higher than $\alpha=3.5^{\circ}$. It is seen that the LW and HW modes have different behaviors with respect to the angle of attack. The LW mode is the first to become unstable and it remains the most unstable up to $\alpha=3.5^{\circ}$. For higher values of angle of attack $\left(\alpha>3.5^{\circ}\right)$, the growth rate of the HW mode strongly increases and quickly dominates the dynamics, so that above $\alpha \geqslant 4^{\circ}$, the growth rate only exhibits a single peak, located at high wavenumber. The peak associated with the LW mode therefore disappears for $\alpha \geqslant 4^{\circ}$. The behavior of the HW mode described in [24] is different: It becomes unstable prior to the LW mode as $\alpha$ increases and it almost always dominates the LW mode for all values of $\alpha$ that they consider. Though for values of $\alpha \leqslant 3.2^{\circ}$ they do find a peak around $\beta \approx 2 \pi$, this peak is much lower than the one existing at high wavenumber. But, as mentioned in Sec. IV D, only a low-wavenumber $(\beta<3 \pi)$ dynamics is experimentally observed for similar flow configurations, which is consistent with the findings of the present article.

Note also that, although the same turbulence models are used in both studies, there are strong differences in the numerical treatment: In the present study, we linearize the discrete URANS equations while Crouch et al. first linearize the continuous equations before discretizing them.

The advantages and drawbacks of each approach are discussed in [37]: In particular, strong advantages of the discretize-then-linearize approach are that it automatically ensures consistency between stability and URANS computations and no ad hoc treatment of the shock-wave region and of the boundary conditions (as done in [24]) is required to carry out linear stability analyses. Nonetheless, it is unclear at this point why such differences are observed and why these only affect the HW mode and not also the LW one.

\section{F. Onset}

Section IV E provides information about the onset of the 3D unstable modes for $\Lambda=0^{\circ}$. Indeed, Fig. 10 shows that the LW buffet cell modes become unstable when increasing $\alpha$ from $3.0^{\circ}$ to $3.1^{\circ}$. The same analysis has been done for $\Lambda \neq 0^{\circ}$ and revealed a slight effect of the sweep angle on the critical value of $\alpha$. Figure 11 shows that for $\Lambda=30^{\circ}$, the 3D mode becomes unstable for $\alpha$ between $3.1^{\circ}$ and $3.2^{\circ}$. The shift of $0.1^{\circ}$ stems from the stabilizing effect of sweep, which was already mentioned in Sec. IV D [see Fig. 9(a)]. Another difference with respect to the $\Lambda=0^{\circ}$ case is that for the swept wing, the frequency of the $3 \mathrm{D}$ modes is no longer zero. Thus, when $\Lambda \neq 0^{\circ}$, the instability is the result of a Hopf bifurcation, while it was of pitchfork type in the case $\Lambda=0^{\circ}$. Finally, regarding the 2D buffet mode, a similar analysis at $\beta=0$ shows that for the unswept case, it becomes unstable when increasing $\alpha$ from $2.9^{\circ}$ to $3.0^{\circ}$ through a Hopf bifurcation [Fig. 12(a)], in accordance with the results from [6]. Like the 3D mode, if $\Lambda$ is set to nonzero values, the onset of the $2 \mathrm{D}$ buffet mode is slightly shifted toward higher values of $\alpha$ due to the stabilizing effect of sweep [Fig. 12(b)]. 

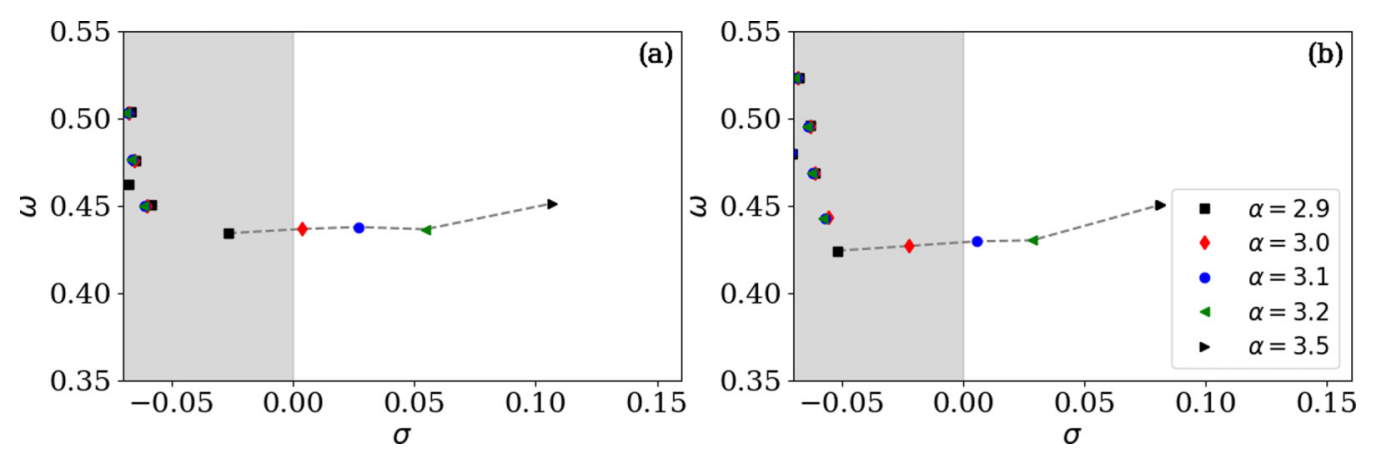

FIG. 12. Spectrum evolution for $\beta=0$ with the angle of attack $\alpha$ increasing from $2.9^{\circ}$ to $3.5^{\circ}$ : (a) unswept wing at $\Lambda=0^{\circ}$ and (b) swept wing at $\Lambda=30^{\circ}$. 2D buffet mode path is outlined by dashed lines.

To conclude, the onset of the 2D and 3D LW modes are very close and an interplay between the two modes could exist close to onset. For this, coupled nonlinear amplitude equations [38] could be derived to analyze in detail the nonlinear dynamics. Another interesting point observed for both unswept and swept wings is that both modes are unstable only when the boundary layer is separated. Also, the growth rate of the LW 3D mode is seen to be slightly more sensitive to $\alpha$ than the $2 \mathrm{D}$ buffet mode (larger variations of $\sigma$ with $\alpha$ ).

\section{G. Adjoint problem and structural sensitivity}

The results of the adjoint problem (10) are presented in this section together with the structural sensitivity. Figure 13 shows the $2 \mathrm{D}$ adjoint buffet mode for the unswept wing at $\alpha=3.5^{\circ}$. As already explained, the 2D mode is presented for $\beta=0$. The spatial structure of the mode in Fig. 13 is similar to the adjoint mode from Sartor et al. [8] for 2D buffet. The region where the values of the mode are the highest are localized in the attached boundary layer and the recirculation bubble on the suction side of the wing and along the characteristic line inside the supersonic zone impacting the shock foot. The 2D adjoint mode of the swept wing is not presented here because, as already mentioned for the direct mode, no relevant differences exist with respect to the adjoint mode at zero sweep (only the appearance of nonzero and constant values of $\rho w^{\dagger}$ in span). Figures 14(a) and 14(b) show the adjoint mode for the unstable 3D mode at $\Lambda=0^{\circ}$ and $\Lambda=30^{\circ}$. Both adjoint modes show in the $x-y$ plane a shape similar to the 2D mode (the characteristic lines inside the supersonic zone are even more visible) and a 3D pattern in span. The highest values are localized at the shock

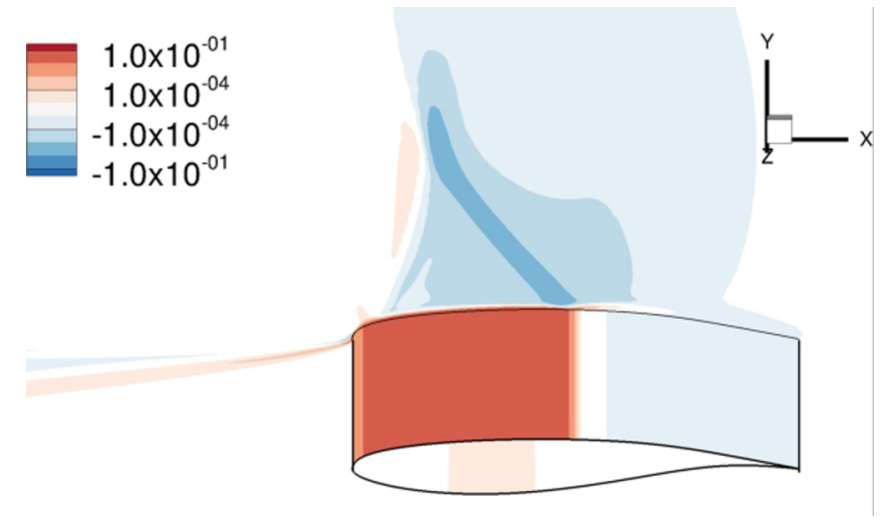

FIG. 13. Real part of the $\rho E$ component of the $2 \mathrm{D}$ buffet adjoint mode at $\beta=0$ for the unswept wing at $M=0.73$ and $\alpha=3.5^{\circ}$. Note the exponential color bar. 


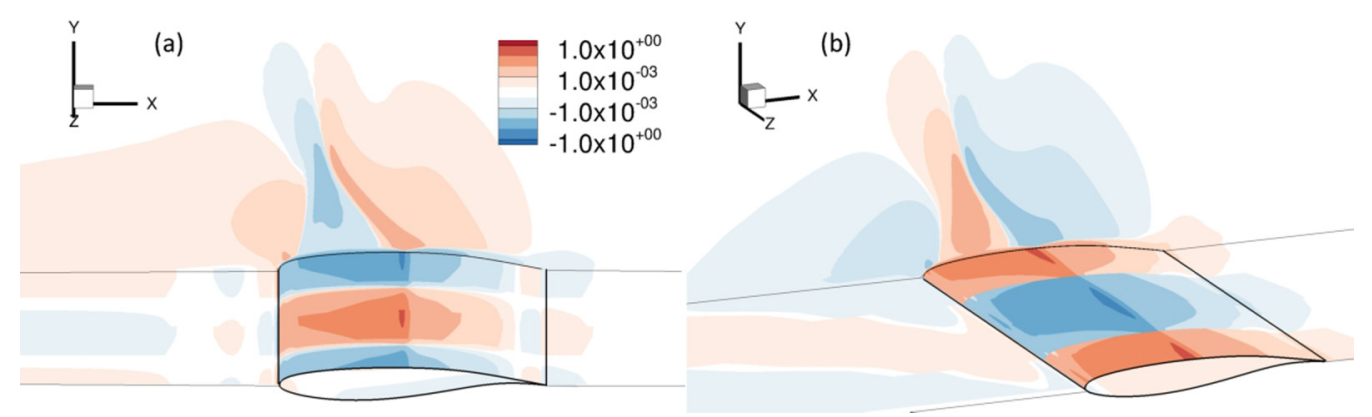

FIG. 14. Real part of the $\rho E$ component of the 3D buffet adjoint mode at $\beta=2 \pi, M=0.73$, and $\alpha=3.5^{\circ}$ for (a) the unswept wing at $\Lambda=0^{\circ}$ and (b) the swept wing at $\Lambda=30^{\circ}$. Note the exponential color bar and the three surfaces where the modes are shown: Wing body, iso- $z$, and iso- $y$ upstream and downstream of the airfoil.

foot and they spread more upstream than downstream, in accordance with the adjoint nature of this mode. Looking at the $y=0$ plane upstream of the airfoil, it is noticed that the isolines of the adjoint mode are aligned with the direction of the freestream velocity. An adjoint mode depicts the region in space where an external forcing has the strongest effect on the unstable global mode and, consequently, adjoint modes are important to define control strategies [32,39]. Indeed, flow control consists in acting on the flow either to suppress a given dynamics or, if this is not possible, to shift its frequency. The present findings show that the region of highest sensitivity is, for both the $2 \mathrm{D}$ and 3D modes, the shock foot, the attached boundary layer, and the characteristic line in the supersonic region. Therefore, it may be possible to define an efficient control strategy for both the $2 \mathrm{D}$ and $3 \mathrm{D}$ transonic buffet modes since they share the same high-sensitivity regions.

The computation of both direct and adjoint modes allows to perform a further analysis of the instability. The direct mode underlines the coherent oscillation of the instability while the adjoint mode highlights its most sensitive region. The region of the flow where the two modes overlap defines the core of the instability. Huerre and Monkewitz [40] first introduced the idea of a region in space where the instability waves are intrinsically generated, called the wavemaker. Several definitions of wavemaker have been proposed and, today, the most accepted one comes from Giannetti and Luchini [41]. They defined the wavemaker as a structural sensitivity that quantifies how an eigenvalue is affected by the introduction of localized forcing. Consequently, in the region of highest values of the wavemaker, a localized feedback in the equations governing a global mode leads to the largest shift of its eigenvalue. Here we only consider localized feedbacks in the linearized momentum equations (and not in the density and/or eddy-viscosity equations), which may be accounted for in the wavemaker formalism with diagonal matrices $P_{j}$ associated with the $j$ th cell, defined in the following way: All diagonal components of $P_{j}$ are zero except for the momentum components of the $j$ th cell where they are equal to 1 . The wavemaker of the $j$ th cell is then obtained following

$$
S_{j}=\left\|P_{j} \mathbf{q}^{\dagger}\right\|_{2} \cdot\left\|P_{j} \hat{\mathbf{q}}\right\|_{2} \quad \text { with } \quad\left\langle\mathbf{q}^{\dagger}, \hat{\mathbf{q}}\right\rangle=1,
$$

where $\|\cdot\|_{2}$ refers to the 2-norm of a vector and $\langle\cdot, \cdot\rangle$ is the scalar product defined in Eq. (7).

The vector $\mathbf{S}$, whose components are the local values $S_{j}$, can then be plotted for both $2 \mathrm{D}$ and $3 \mathrm{D}$ modes. Figure 15 shows the structural sensitivity of the 2D buffet mode. It shows the same structure of the wavemaker presented by Iorio et al. [21]. The core of the 2D instability is the shock foot with lower values in the shock and in the detached boundary layer. Figures 16(a) and 16(b) show the structural sensitivity of the 3D mode for the unswept wing and swept wing at $\Lambda=30^{\circ}$, respectively. The real and imaginary parts of both the direct and the adjoint 3D modes exhibit a harmonic shape in $z$ and are shifted by $90^{\circ}$. This is the reason why the norm of the modes is constant in the spanwise direction, and therefore also the wavemaker. 


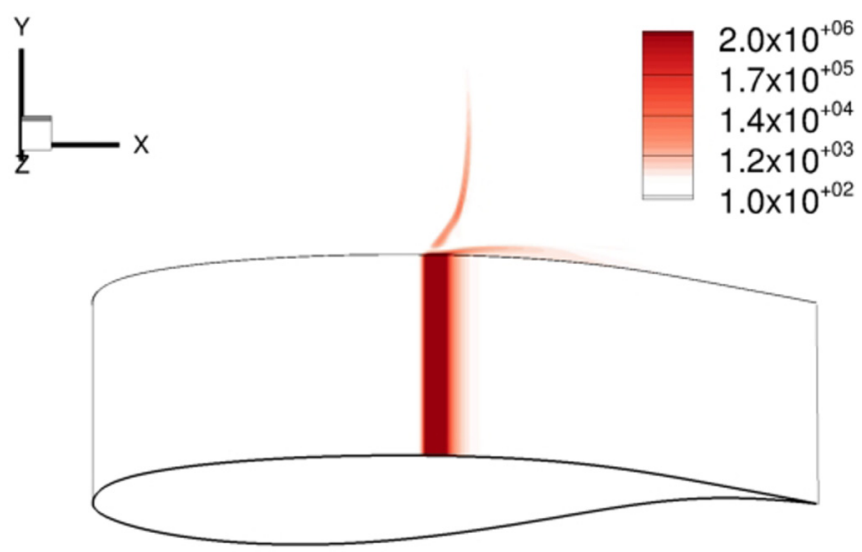

FIG. 15. Structural sensitivity $\mathbf{S}$ of the 2D mode at $\beta=0$ for the unswept wing at $M_{n}=0.73$ and $\alpha=3.5^{\circ}$. The color bar is exponential.

The separation line, which also corresponds to the shock foot location, is the region with the highest values of structural sensitivity but the separated region also exhibits high values. The core of the 3D buffet cell mode is in fact localized closer to the wing by comparison with the 2D instability: The wavemaker extends further in the separated region (see skin values in Figs. 15 and 16) and is limited to the very near-wall region of the shock (see $z$ plane values in Figs. 15 and 16). The analysis of the wavemaker therefore indicates that the instability is really linked to the separation region and not so much to the shock. The presence of a nonzero sweep angle does not remarkably influence the shape of the structural sensitivity.

\section{SUMMARY AND CONCLUSION}

The evidence that the transonic buffet phenomenon on 2D airfoils is due to a global unstable mode was first shown by Crouch et al. [6]. It has been largely confirmed in past years [8,21]. In the present study, a 3D global stability analysis has been carried out on an infinite wing in transonic flow conditions. The objective was to find a link between the $2 \mathrm{D}$ transonic buffet global mode and the 3D transonic buffet for which frequencies are four to seven times higher [12]. The present results have shown that when perturbations are allowed to be 3D, there are actually two unstable modes: A 2D one identical to the one discovered by Crouch et al. and a 3D LW mode. The latter exhibits zero frequency for an unswept wing and becomes unsteady when the sweep angle increases. Its growth rate is higher than the $2 \mathrm{D}$ mode and its frequency increases linearly with the sweep angle, up to six times the $2 \mathrm{D}$ one at $30^{\circ}$, which explains why the buffet frequency is much higher in three
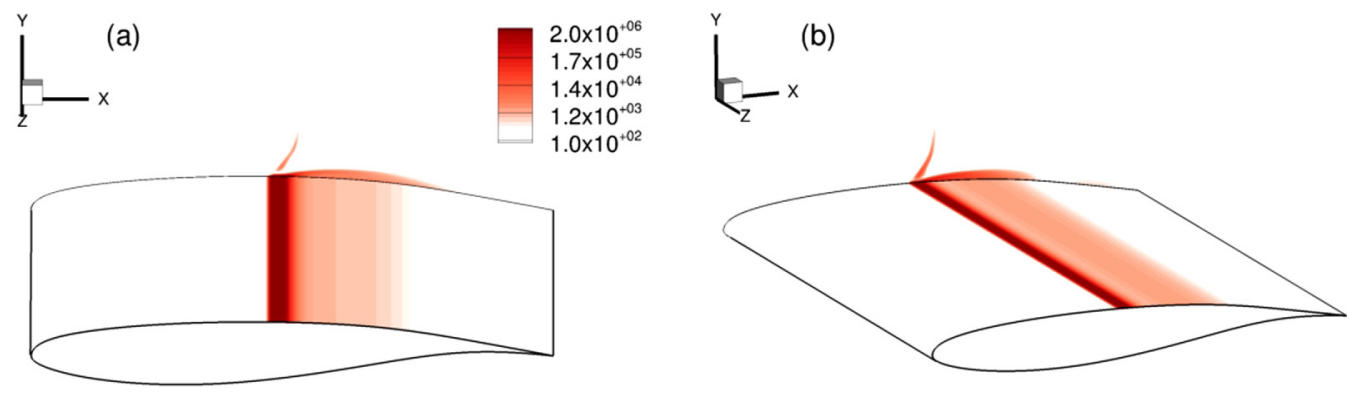

FIG. 16. Structural sensitivity $\mathbf{S}$ of the 3D mode at $\beta=2 \pi, M_{n}=0.73$, and $\alpha=3.5^{\circ}$ for (a) the unswept wing at $\Lambda=0^{\circ}$ and (b) the swept wing at $\Lambda=30^{\circ}$. The color bar is exponential. 
than in two dimensions. This 3D LW mode corresponds to the buffet cell phenomenon observed by Iovnovich and Raveh [14]. The wavenumber $(\beta \approx 2 \pi$ or a wavelength equal to one chord length) and the convection velocity $\left(0.43 U_{n \infty}\right.$ or $\left.0.37 U_{\infty}\right)$ of this $3 \mathrm{D}$ mode are in very good agreement with what has been observed experimentally [12,18] and numerically [15].

When looking at higher angles of attack, the 3D LW buffet mode at $\beta \approx 2 \pi$ is no longer the leading unstable mode. Another $3 \mathrm{D}$ mode appears at $\beta \approx 30$ that exhibits an even higher growth. This 3D HW mode is the leading one only at high angles of attack and low sweep angles, which is presumably the reason why it has not been observed in experiments until now. Yet, the 3D HW mode has been shown to behave differently with respect to the results from Crouch et al. [24]: For example, the 3D HW mode nearly always dominates the 3D LW mode in [24] while this is only the case in our study at high angles of attack and low sweep angles. The analysis of the adjoint modes shows similar regions of optimal forcing for both 2D and 3D modes. Finally, the structural sensitivities highlight the core of the instabilities which is for both modes localized near the shock foot with higher values of sensitivity in the detached boundary layer for the 3D mode.

The main conclusion of the present study is that the phenomenon called in the literature 3D transonic buffet corresponds to a buffet cell convection phenomenon with a zero-frequency unstable global mode which becomes unsteady with sweep. The mode also appears to be distinct from the dynamics of the 2D mode. Hence, the analysis of the wavemaker of the 3D mode reveals that the core of the instability is nearly solely in the separated region (and only very weakly along the shock), with a maximum along the separation line. This is in contrast with the 2D mode for which the shock plays a greater role, since the wavemaker for this latter mode also exhibits strong values along the shock wave.

A future perspective of the present study is to improve the numerical method in order to analyze more complex $3 \mathrm{D}$ wings. It will be interesting to analyze the effect of the boundary conditions in span as well as the addition of a taper ratio and twist on the buffet frequency.

[1] B. H. K. Lee, Oscillatory shock motion caused by transonic shock boundary-layer interaction, AIAA J. 28, 942 (1990).

[2] A. Hartmann, A. Feldhusen, and W. Schröder, On the interaction of shock waves and sound waves in transonic buffet flow, Phys. Fluids 25, 026101 (2013).

[3] L. Jacquin, P. Molton, S. Deck, B. Maury, and D. Soulevant, Experimental study of shock oscillation over a transonic supercritical profile, AIAA J. 47, 1985 (2009).

[4] S. Deck, Numerical simulation of transonic buffet over a supercritical airfoil, AIAA J. 43, 1556 (2005).

[5] Q. Xiao, H. M. Tsai, and F. Liu, Numerical study of transonic buffet on a supercritical airfoil, AIAA J. 44, 620 (2006).

[6] J. D. Crouch, A. Garbaruk, and D. Magidov, Predicting the onset of flow unsteadiness based on global instability, J. Comput. Phys. 224, 924 (2007).

[7] J. D. Crouch, A. Garbaruk, D. Magidov, and A. Travin, Origin of transonic buffet on aerofoils, J. Fluid Mech. 628, 357 (2009).

[8] F. Sartor, C. Mettot, and D. Sipp, Stability, receptivity, and sensitivity analyses of buffeting transonic flow over a profile, AIAA J. 53, 1980 (2015).

[9] F.W. Roos, The buffeting pressure field of a high-aspect-ratio swept wing, AIAA Paper No. 1985-1609 (1985).

[10] J. Reneaux, V. Brunet, D. Caruana, S. Deck, and P. Naudin, A combined experimental and numerical investigation of the buffet phenomenon and its control through passive and active devices, in Proceedings of the CEAS Katner Conference on Key Aerodynamic Technologies, Bremen, Germany (2005).

[11] P. Molton, J. Dandois, A. Lepage, V. Brunet, and R. Bur, Control of buffet phenomenon on a transonic swept wing, AIAA J. 51, 761, (2013).

[12] J. Dandois, Experimental study of transonic buffet phenomenon on a 3D swept wing, Phys. Fluids 28, 016101 (2016). 
[13] S. Koike, M. Ueno, K. Nakakita, and A. Hashimoto, Unsteady pressure measurement of transonic buffet on nasa common research model, AIAA Paper No. 2016-4044 (2016).

[14] M. Iovnovich and D. E. Raveh, Numerical study of shock buffet on three-dimensional wings, AIAA J. 53, 449 (2015).

[15] F. Plante, J. Dandois, F. Sartor, and E. Laurendeau, Study of three-dimensional transonic buffet on swept wings, in Proceedings of the 35th AIAA Applied Aerodynamics Conference (AIAA, Washington, DC, 2017), p. 3903.

[16] V. Brunet and S. Deck, Zonal-detached eddy simulation of transonic buffet on a civil aircraft type configuration, AIAA Paper No. 2008-4152 (2008).

[17] F. Sartor and S. Timme, Delayed detached-eddy simulation of shock buffet on half wing-body configuration, AIAA J. 55, 1230 (2016).

[18] Y. Sugioka, S. Koike, K. Nakakita, D. Numata, T. Nonomura, and K. Asai, Experimental analysis of transonic buffet on a 3D swept wing using fast-response pressure-sensitive paint, Exp. Fluids 59, 108 (2018).

[19] D. Rodríguez and V. Theofilis, On the birth of stall cells on airfoils, Theor. Comput. Fluid Dyn. 25, 105 (2011).

[20] R. Gioria, W. He, J. M. Pérez, and V. Theofilis, Modal and non-modal global instability analyses of low-Re massively separated flow around a NACA 0015 airfoil, in Proceedings of the 46th AIAA Fluid Dynamics Conference (AIAA, Washington, DC, 2016), p. 3778.

[21] M. C. Iorio, L. M. Gonzalez, and E. Ferrer, Direct and adjoint global stability analysis of turbulent transonic flows over a naca0012 profile, Int. J. Numer. Methods Fluids 76, 147 (2014).

[22] S. Timme and R. Thormann, Towards three-dimensional global stability analysis of transonic shock buffet, AIAA Paper No. 2016-3848 (2016).

[23] S. Timme, Global instability of wing shock buffet, arXiv:1806.07299.

[24] J. D. Crouch, A. Garbaruk, and M. Strelets, Global instability analysis of unswept- and swept-wing transonic buffet onset, AIAA Paper No. 2018-3229 (2018).

[25] P. J. Schmid, M. F. de Pando, and N. Peake, Stability analysis for $n$-periodic arrays of fluid systems, Phys. Rev. Fluids 2, 113902 (2017).

[26] P. R. Spalart and S. R. Allmaras, A one equation turbulence model for aerodynamic flows, AIAA Paper No. $1992-0439$ (1992).

[27] P. R. Spalart, Trends in turbulence treatments, AIAA Paper No. 2000-2306 (2000).

[28] I. Mary and P. Sagaut, Large eddy simulation of flow around an airfoil near stall, AIAA J. 40, 1139 (2002).

[29] M. Pechier, P. Guillen, and R. Cayzac, Magnus effect over finned projectiles, J. Spacecr. Rockets 38, 542 (2001).

[30] J.-M. Chomaz, Global instabilities in spatially developing flows: Non-normality and nonlinearity, Annu. Rev. Fluid Mech. 37, 357 (2005).

[31] O. Marquet, M. Lombardi, J.-M. Chomaz, D. Sipp, and L. Jacquin, Direct and adjoint global modes of a recirculation bubble: Lift-up and convective non-normalities, J. Fluid Mech. 622, 1 (2009).

[32] D. Sipp, Open-loop control of cavity oscillations with harmonic forcings, J. Fluid Mech. 708, 439 (2012).

[33] S. Beneddine, Characterization of unsteady flow behavior by linear stability analysis, Ph.D. thesis, Université Paris-Saclay (2017).

[34] R. B. Lehoucq, D. C. Sorensen, and C. Yang, ARPACK Users' Guide: Solution of Large-Scale Eigenvalue Problems with Implicitly Restarted Arnoldi Methods (Society for Industrial and Applied Mathematics, Philadelphia, PA, 1998), Vol. 6.

[35] P. R. Amestoy, I. S. Duff, J.-Y. L'Excellent, and J. Koster, A fully asynchronous multifrontal solver using distributed dynamic scheduling, J. Matrix Anal. Appl. 23, 15 (2001).

[36] L. Manni, T. Nishino, and P.-L. Delafin, Numerical study of airfoil stall cells using a very wide computational domain, Comput. Fluids 140, 260 (2016).

[37] C. Mettot, F. Renac, and D. Sipp, Computation of eigenvalue sensitivity to base flow modifications in a discrete framework: Application to open-loop control, J. Comput. Phys. 269, 234 (2014).

[38] P. Meliga, J.-M. Chomaz, and D. Sipp, Global mode interaction and pattern selection in the wake of a disk: A weakly nonlinear expansion, J. Fluid Mech. 633, 159 (2009). 
[39] D. Sipp, O. Marquet, P. Meliga, and A. Barbagallo, Dynamics and control of global instabilities in openflows: A linearized approach, Appl. Mech. Rev. 63, 030801 (2010).

[40] P. Huerre and P. A. Monkewitz, Local and global instabilities in spatially developing flows, Annu. Rev. Fluid Mech. 22, 473 (1990).

[41] F. Giannetti and P. Luchini, Structural sensitivity of the first instability of the cylinder wake, J. Fluid Mech. 581, 167 (2007). 